\title{
ARTICLE OPEN \\ Accelerated aging test modeling applied to solar mirrors
}

\author{
Coralie Avenel $\mathbb{D D}^{1,2}$, Olivier Raccurt ${ }^{1}$, Jean-Luc Gardette ${ }^{2}$ and Sandrine Therias ${ }^{2}$
}

The durability of solar mirrors is a critical factor for the deployment of concentrating solar power plants. Accelerated aging test models currently applied in the polymer, electronic, and photovoltaic fields have recently been reviewed, and the issues of their application to solar mirrors have been discussed. This article first reports the results of temperature, humidity, and light irradiance accelerated aging tests performed to assess the dependent parameters of selected models from the literature. These parameters include the apparent activation energy for the Arrhenius temperature law, the Peck and Eyring coefficients for humidity models and the Schwarzschild coefficient for the irradiance law. The experimental values were then assessed for specular reflectance loss of solar mirrors. Finally, using these parameters, acceleration factors were calculated for solar mirrors. An effective temperature considering the Arrhenius degradation law was used rather than the commonly used mean temperature. This question is also addressed for light irradiance by using the dose instead of the mean value.

npj Materials Degradation (2019)3:27; https://doi.org/10.1038/s41529-019-0089-y

\section{INTRODUCTION}

Solar energy is a renewable and carbon dioxide-free way to produce energy. Several technologies exist, including photovoltaic (PV), concentrated photovoltaic (CPV), thermal, and concentrated solar power (CSP) systems. In CSP and CPV plants, sunlight is concentrated by mirrors on an absorber to heat a fluid or by Fresnel lenses on the PV module, respectively. Due to the elevated costs of technologies in the solar field, solar mirrors must retain their functional properties during the whole life of the plant, which is expected to be longer than 25-30 years. ${ }^{1}$ Consequently, durability is one of the main issues in bringing products to the market. Durability can be defined in many ways, but there is consensus that it corresponds to the period of time a product in its service environment will survive before requiring replacement or maintenance. In other words, the time to failure is defined as the time before a functional property of the material decreases under the minimum requirement for the application. The weathering resistance of mirrors exposed to all environmental and operational stresses should then be evaluated to predict whether or not a technology is adapted to an implantation site.

Environmental stresses are defined by the potential locations for application. As CSP and CPV plants only use direct normal irradiance (DNI), they must be installed in specific locations that fulfill several requirements, such as high DNI, large plain land, etc. Potential locations on Earth for CSP have been determined (SolarPACES. Concentrating Solar Power Projects http://www.nrel. gov/csp/solarpaces (accessed April 15, 2018)). According to the Köppen-Geiger climate classification, ${ }^{2}$ these locations are in arid regions or areas near the sea that have aggressive stress factors: sunlight, high and cycling temperature, humidity, rain, saline mist, wind, sand storms, etc.

Solar mirrors have been subjected to long-term durability studies to determine the most relevant stress factors, which have been identified as temperature, irradiance, and humidity. ${ }^{3-6}$ As these stresses reach high levels in the locations previously identified, most exposed materials are susceptible to undergo a rapid degradation of their properties.

As natural aging can be far too long considering the 30-years expected lifetime ${ }^{1}$ of materials, accelerated testing and service life prediction are important in judging whether materials will be suitable for applications. The goal of any accelerated weathering test is to increase the rate of degradation of material performance to obtain information much faster than under service conditions. However, accelerated testing and service life prediction require knowledge of the environmental stresses and how the material will respond. Natural exposure on representative sites is always needed, because the relevance of accelerated tests must be confirmed, and the degradation mechanism in a defined accelerated aging test must be verified to be identical to that occurring under service conditions. Methodologies for designing accelerated tests and making service life predictions can be found in the literature. ${ }^{7}$ Current progress in different steps of solar mirror development has recently been summarized. ${ }^{6}$

Once the accelerated test has been qualified as relevant, its acceleration factor $A$ can be calculated in relation to a given site. The acceleration factor is defined as the relationship between the time to failure in service $t_{s}$ and the time to failure in test $t_{\text {test }}$ leading to the same degradation, as given by Eq. (1):

$A=\frac{t_{s}}{t_{\text {test }}}$

The time to failure in service is the lifetime of the product that this methodology aims to determine and is obviously unknown. The time to failure must consequently be linked to the physicochemical process of degradation, which depends on several stress factors on site. A relationship can then be established between this time and the stress load, and the acceleration factor is calculated using the loads in the aging test and on the application site.

\footnotetext{
${ }^{1}$ Univ. Grenoble Alpes - CEA, LITEN, Thermal, Biomass and Hydrogen Department, F-38054 Grenoble, France and ${ }^{2}$ Univ. Clermont Auvergne - CNRS - SIGMA Clermont, ICCF, F-63000 Clermont-Ferrand, France

Correspondence: Olivier Raccurt (olivier.raccurt@cea.fr) or Sandrine Therias (sandrine.therias@uca.fr)
}

Received: 19 February 2019 Accepted: 26 June 2019

Published online: 12 July 2019 
Table 1. Meaning of symbols used in equations

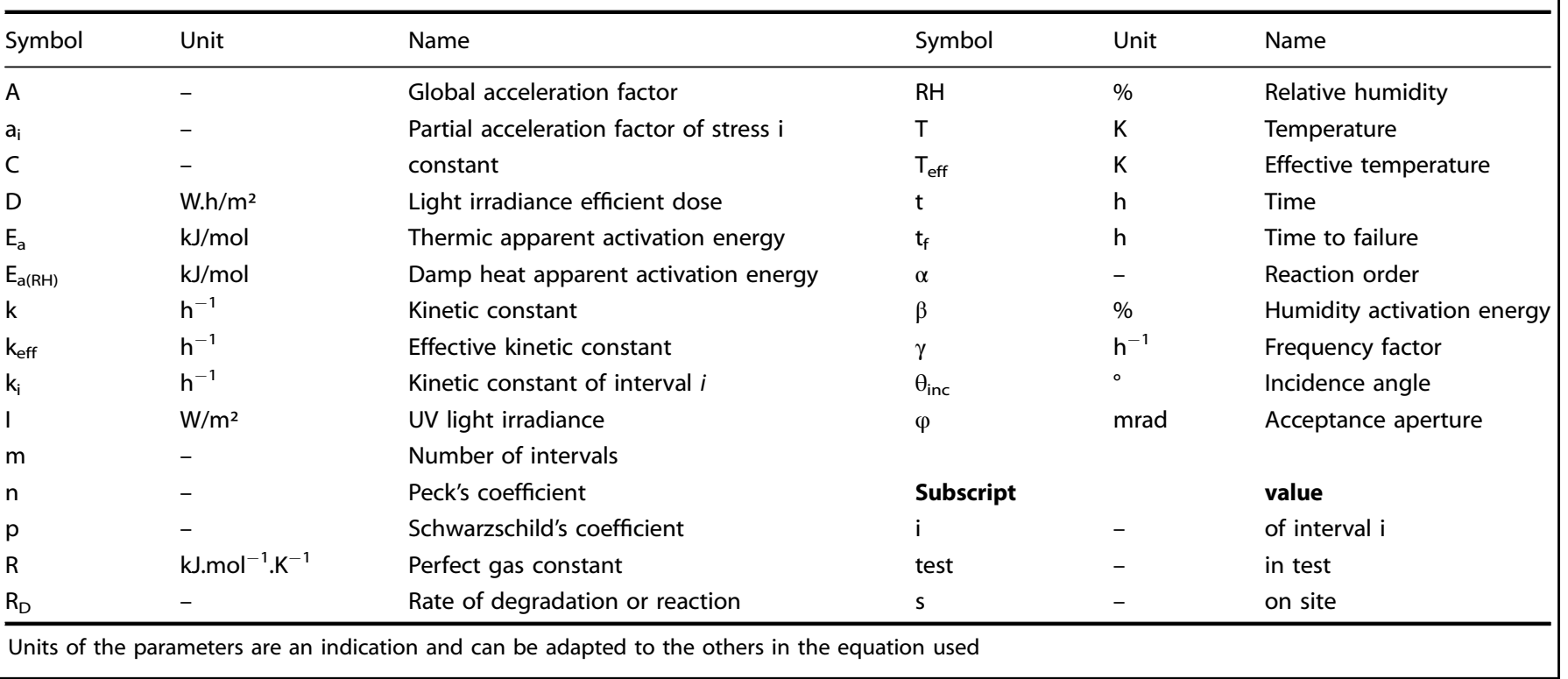

To simplify the rather complex phenomena occurring on site and to make a first approach toward lifetime prediction, stress factors should be considered independently of each other. Consequently, the accelerated tests designed for this method and presented in this article take into account only one stress factor and are performed at a constant value. An exception is temperature, which can also be considered as an accelerator rather than a stress factor in its own right. Consequently, models for degradation under humidity often also include the temperature level. This point will be discussed further for the concerned models.

To understand the degradation mechanisms, the composition of the solar mirror must first be analyzed. Mirrors can be classified into three main types: glass, aluminum, and polymer mirrors. ${ }^{8,9}$ Several plants have been installed and exploited since $1980^{10,11}$ using glass monolithic mirrors, and little degradation of the mirrors has been observed. However, mirror technologies have since evolved, and new mirrors on the market differ from the ones installed on these plants. ${ }^{12}$ In particular, the previous system of paints for monolithic mirrors contained large amounts of lead to improve stability, but recent environmental regulations have demanded a drastic decrease in lead content or even its total removal.

The binder in such paints is a polymer or mix of polymers that bind together other elements, such as inorganic fillers and pigments that have the desired protective properties, such as anti-UV, oxygen, and water barrier properties. It is, therefore, relevant to approach the study of their lifetime using the methodology and models developed in the field of polymer degradation. Another issue is that since all solar technologies face relatively identical environmental conditions, it also seems relevant to use models established for solar fields, such as for photovoltaic and solar thermal technologies. The relationships used in these fields to model the effect of environmental stress factors have recently been reviewed, and their application to solar mirrors has also been discussed. ${ }^{7}$

All the accelerated tests were designed in order to determine kinetic parameters of the previously selected numerical models, and to assess the relevance of these models for solar mirrors lifetime prediction. Tests were performed on three commercially available glass mirrors from different manufacturers, named $A, B$, and $C$ to keep confidentiality. The specular reflectance of each sample was monitored at several times of test, and experimental results are presented hereafter as the specular reflectance loss as a function of test time.

Kinetic parameters calculated from experimental results were then used to make a numerical assessment of acceleration factors. An acceleration factor assessment can only be done compared to a define location, so 9 CSP application sites with different meteorological conditions were selected as examples. Meteorological data were supplied by Meteonorm 7.2 software (Company, Meteotest Meteonorm 7: Global Meteorological Database for Solar Energy and Applied Climatology. (2015)).

This is a brief presentation of the methodology leading to the following results, detailed information can be found in the "Methods" section. In order to make the reading easier, a table (see Table 1) summarizes the nomenclature used in the article.

\section{RESULTS AND DISCUSSION}

Basic considerations on Arrhenius relationship

Temperature is a common stress that accelerates the degradation of a material because it accelerates kinetic processes. Most experimental data obtained with the temperature stress factor are modeled with the Arrhenius relationship, ${ }^{13-15}$ which assumes that a chemical degradation process is controlled by a reaction rate constant $k$, as given by Eq. (2), where $\gamma$ is the frequency factor, $E_{a}$ is the apparent activation energy $(\mathrm{kJ} / \mathrm{mol}), R$ is the ideal gas constant $(\mathrm{kJ} / \mathrm{mol} / \mathrm{K})$ and $T$ is the temperature $(\mathrm{K})$ :

$$
k=\gamma \cdot \exp \left(\frac{-E_{a}}{R \cdot T}\right)
$$

The apparent activation energy of a material can be determined by performing aging tests at several constant temperatures, then by plotting $\ln \left(t_{\text {test }}\right)$ as a function of $1 / T$ for different temperatures of the test. The result should be a straight line, and its slope will be $E_{a} / R$. To more accurately determine its value, aging tests must be performed at a minimum of three temperatures, and more temperatures are preferable. Thus, a weak interval between the chosen temperatures should be chosen to avoid any changes in the degradation mechanism and, consequently, the corresponding activation energy. Such changes would lead to a break in the slope of $\ln \left(t_{\text {test }}\right)$ vs $1 / T$ or a deviation in the linear regression of the graphical representation. ${ }^{7}$ 

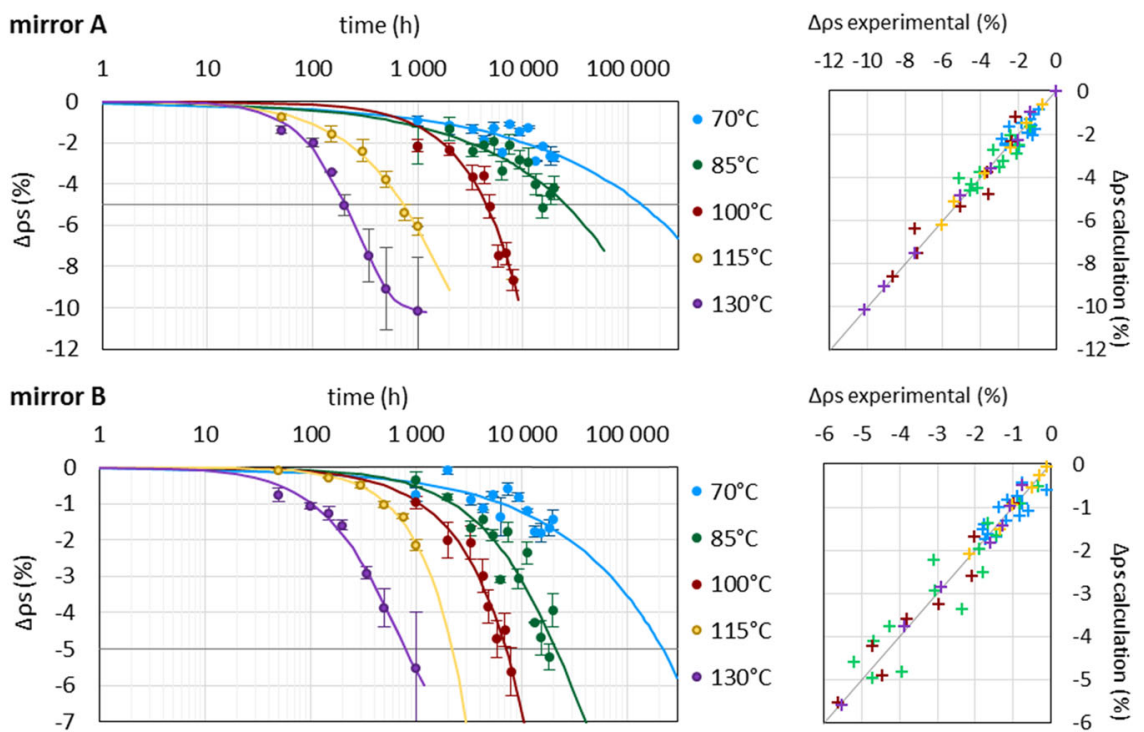

$\Delta \rho$ s experimental (\%)
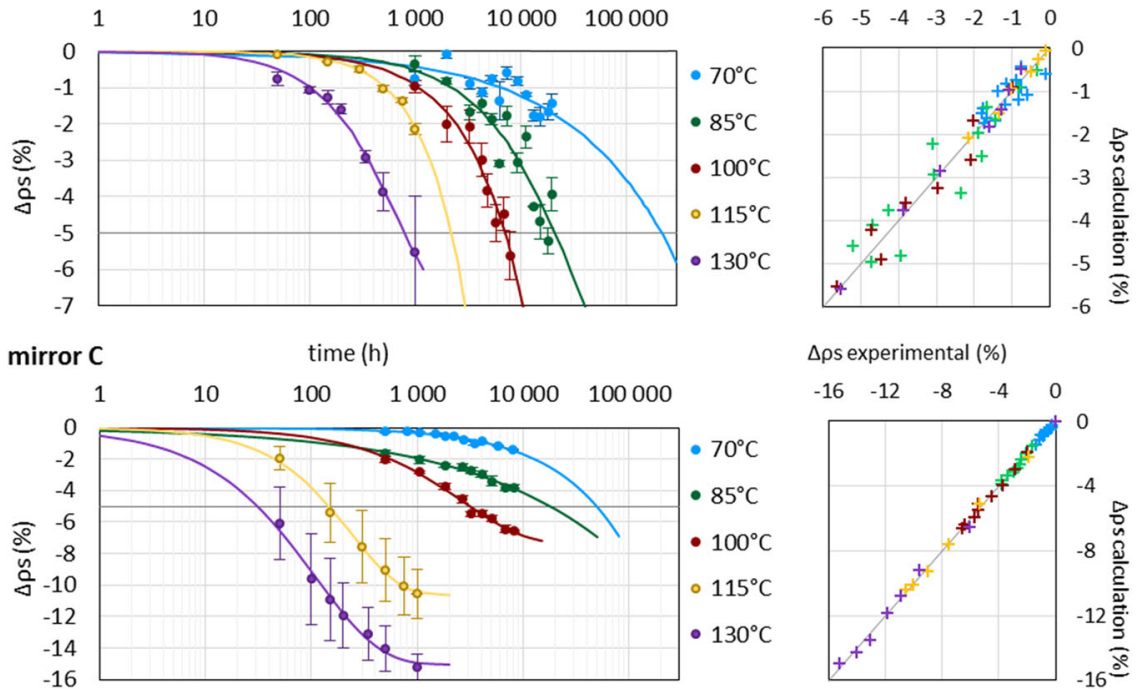

Fig. 1 Specular reflectance loss of mirrors A, B, and C during temperature aging tests (left). The marks are experimental points, and lines are the extrapolation calculated using Eq. (3). The correlation between experimental and calculated points is shown on the right side

Experimental results of mirrors degradation

Tests were performed in the dark at five temperatures between 70 and $130^{\circ} \mathrm{C}$ with a fixed interval of $15^{\circ} \mathrm{C}$. The shape of reflectance loss was not linear with aging time, and thus another type of relationship was investigated to fit the data. The Weibull distribution function ${ }^{16}$ is commonly used to fit nonlinear data and is often used in aging and reliability studies. ${ }^{17-19}$ Consequently, the loss of specular reflectance was extrapolated from experimental measurements by a Weibull distribution function according to Eq. (3):

$\Delta \rho_{s}(t)=K\left[1-\exp \left(-\frac{\left(t .10^{-3}\right)^{a}}{b}\right)\right]$

where $t$ is the time of the test in hours and $K, a$, and $b$ are parameters set to obtain the best fit of the experimental data. In Fig. 1, the experimental data are represented by marks, and the calculated extrapolation obtained using Eq. (3) is represented by lines. The time axis is represented with a log scale to highlight the initiation of degradation.

The correlation between the experimental points and the Weibull equation calculation is presented for all tests. The global tendency is appropriate for all curves, and thus the Weibull function is validated for these data.

Arrhenius numerical model parameter calculation based on experimental results

Equation (2) can be rearranged to give Eq. (4), which facilitates the determination of the apparent activation energy $E_{a}$. Plotting In ( $\left.t_{\text {test }}\right)$ as a function of $1 / T_{\text {test }}$ the product of the slope and $R$ constant gives $E_{a}$. These "Arrhenius representations" are shown in
Fig. 2 for the three mirrors tested.

$$
\ln \left(t_{\text {test }}\right)=\frac{E_{a}}{R} \cdot \frac{1}{T_{\text {test }}}+\ln \left(t_{s}\right)-\frac{E_{a}}{R \cdot T_{s}}
$$

The Arrhenius representation leads to straight lines, thus validating the Arrhenius law for mirror degradation due to temperature. The temperature aging tests permitted the determination of the apparent thermic activation energy of the three solar glass mirrors. The resulting apparent activation energies were 125, 105 , and $141 \mathrm{~kJ} / \mathrm{mol}$ for mirrors $\mathrm{A}, \mathrm{B}$, and $\mathrm{C}$, respectively. All values were in agreement with the order of magnitude of activation energies for polymer degradation. ${ }^{20}$ This finding is relevant because of the paints protecting the silver layer are made with polymer binders. When the paint is degraded by temperature, it can no longer play its barrier role, and then elements are free to diffuse inside until they reach the silver layer.

\section{Effective temperature definition}

The acceleration factor obtained using the Arrhenius relationship is given in Eq. (5), which assumes that the activation energy is constant over the whole range of test and service temperatures.

$a_{T}=\exp \left(\frac{E_{a}}{R}\left(\frac{1}{T_{\text {test }}}-\frac{1}{T_{s}}\right)\right)$

This relationship takes into account fixed values of the test and service temperatures. The test can be performed at a constant temperature; however, natural exposures are subjected to notable temperature variations induced by the alternation between day and night, seasons and other climatic variations. The current and simplest approach consists in using the annual mean temperature on site. Nevertheless, the temperature parameter is included in the exponential factor, which implies that the relationship 


\section{mirror A}
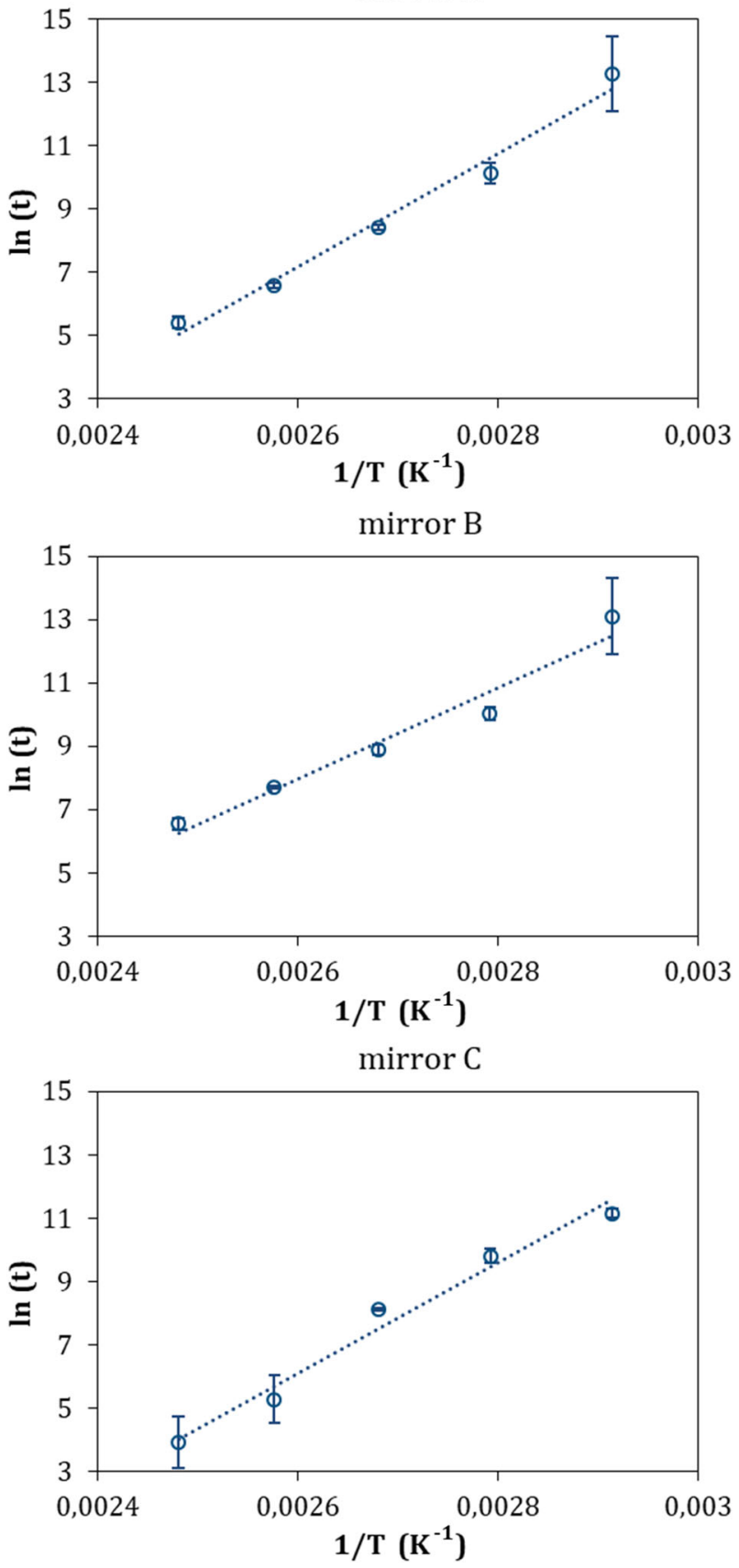

Fig. 2 Graphical representation based on Arrhenius law to determine the apparent activation energy for mirrors $A, B$, and $C$. Error bars represent the standard deviation of experimental measurements

between degradation at high and low temperatures is not linear. To better represent this lack of linearity, high temperatures should play a larger role than low temperatures in calculating a new type of fixed value taking into account the acceleration factor.

Some authors ${ }^{21-23}$ have introduced the concept of an effective temperature. The effective temperature was defined as the temperature that provokes the same degradation of the component exposed to constant temperature rather than to varying temperatures.

It was assumed that the time of exposure could be divided into discrete equal intervals because, in practice, measurements are often obtained at equal intervals of time. Thus, the temperature is

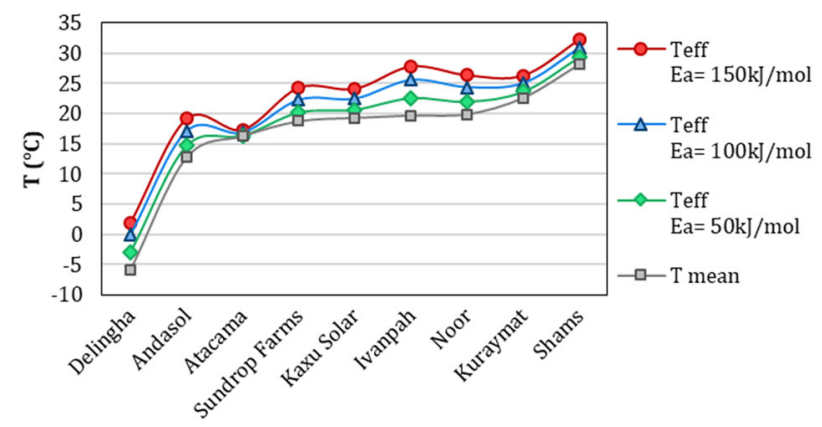

Fig. $3 T_{\text {mean }}$ and $T_{\text {eff }}$ for each site with $E_{a}=50,100$ or $150 \mathrm{~kJ} / \mathrm{mol}$

continuous in a classical climate model, and it is assumed that the rate of degradation follows the Arrhenius equation. In the current article, we used the model proposed by Tencer et al. ${ }^{23}$ They obtain the following Eq. (6):

$$
T_{\text {eff }}=\frac{-E_{a}}{R \ln \left[\frac{1}{t} \int_{0}^{t} \exp \left(\frac{-E_{a}}{R \cdot T}\right) d t\right]}
$$

As this equation depends on the thermal activation energy of the degradation, different materials and component technologies will be associated with different effective temperatures and so different rates of degradation for the same exposure. Thus, the same material can have several effective temperatures because it depends on the failure mechanism of interest, usually the fastest, which can vary according to the exposure conditions. From another point of view, for the same material, the differences between the profiles of temperature variations at different sites will affect the effective temperature. Consequently, the effective temperature must be calculated for each site of interest.

It can be noticed that the effective temperature has already been used in durability studies in the field of solar thermal absorbers $^{24,25}$ and the established standards have also considered the effective temperature (ISO 22975-3:2014: Solar energyCollector components and materials-Part 3: Absorber surface durability-2014).

\section{Comparison between mean and effective temperatures}

The impact of the effective temperature on acceleration factors and lifetime prediction compared to the mean temperature is first discussed for the selected sites. Thus, to show the impact of activation energy on the lifetime prediction, three values were selected in a range relevant for polymeric materials used in solar technologies: 50, 100, and $150 \mathrm{~kJ} / \mathrm{mol}$.

The mean and effective temperatures using these three activation energies were calculated for each site and are presented in Fig. 3. All sites were sorted by their mean temperatures on all the following graphs.

The difference between the effective and the mean temperatures is shown in Fig. 4 (left), using relationship (7):

$\Delta T=T_{\text {eff }}-T_{\text {mean }}$

The effective temperature is always higher than the mean temperature, due to the exponential factor in the Arrhenius law. However, the magnitude of this difference depends on sites, because the frequency-temperature profiles are different. Delingha and Ivanpah have the largest distributions of temperature, which extends in a 49 and $48^{\circ} \mathrm{C}$ range, respectively, and their effective temperature is $\sim 8{ }^{\circ} \mathrm{C}$ higher than the mean for $E_{a}=$ $150 \mathrm{~kJ} / \mathrm{mol}$. In contrast, Atacama has the smallest range of temperature extending within $21^{\circ} \mathrm{C}$, and its effective temperature is only $1{ }^{\circ} \mathrm{C}$ higher than the mean for $E_{a}=150 \mathrm{~kJ} / \mathrm{mol}$. Consequently, as illustrated by Fig. 4 (right), this difference depends on the distribution of temperature at the site and increases with the 

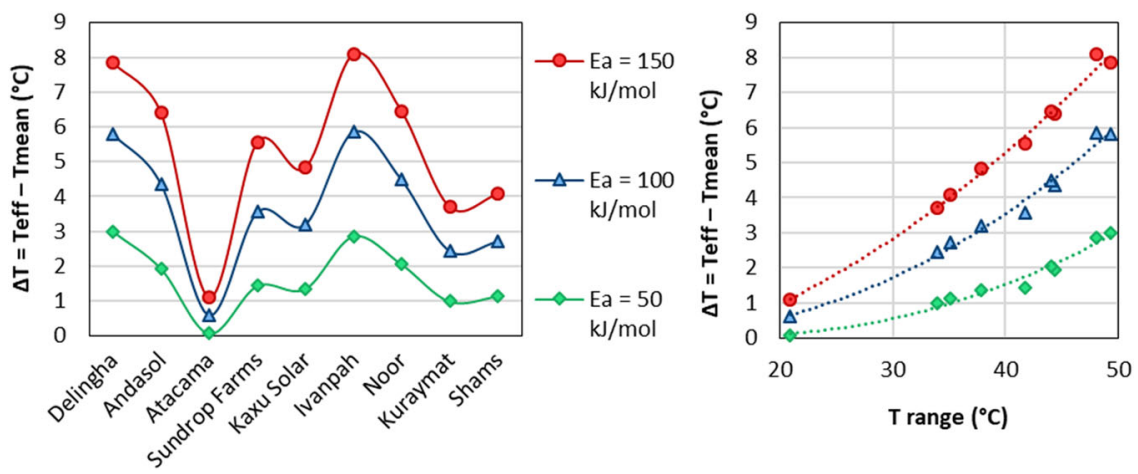

Fig. 4 Difference between the mean and the effective temperatures of the sites for $E_{a}$ between 50 and $150 \mathrm{~kJ} / \mathrm{mol}$, for each site (left) or as a function of the temperature range (right)

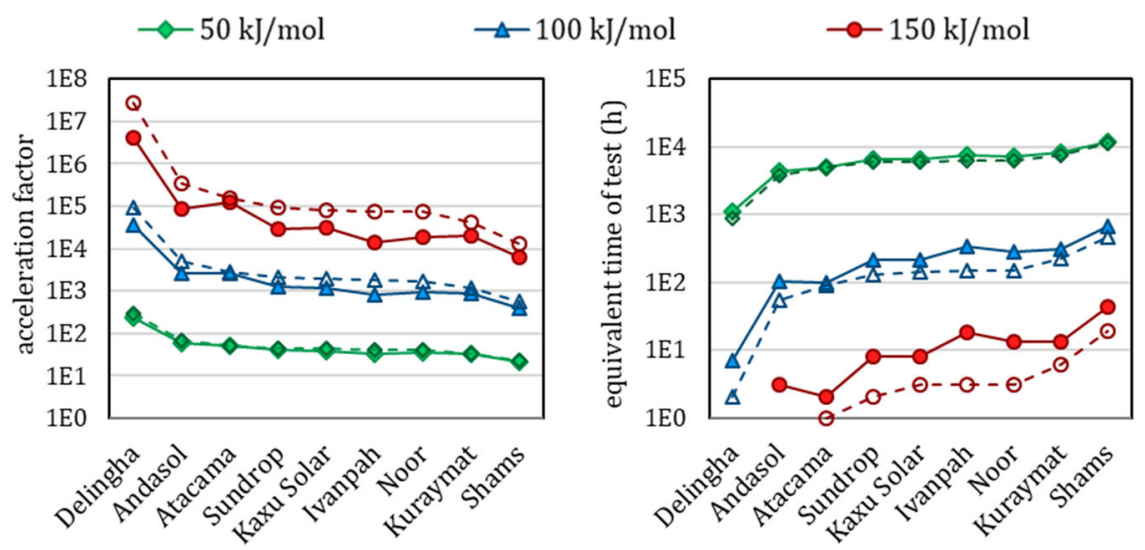

Fig. 5 Acceleration factor (left) and time of test (right) at $85^{\circ} \mathrm{C}$ equivalent to 30 years on 9 sites, calculated with $E_{a}=50,100$ or $150 \mathrm{~kJ} / \mathrm{mol}$ using the effective (full lines) or the mean (dash lines) temperature. Sites are sorted by increasing annual mean temperature

temperature range. This relation seems to follow a polynomial law that obviously depends on the activation energy.

Acceleration factor calculation from the numerical model Three activation energies were chosen as examples in a range including the three previous results: 50,100 , and $150 \mathrm{~kJ} / \mathrm{mol}$. The aging test temperature was set as $T_{\text {test }}=85^{\circ} \mathrm{C}$, since this is the temperature used in the damp heat test for PV devices ${ }^{22}$ or as the maximum temperature in cycling tests. ${ }^{26}$ The lifetime on site was set to $t_{s}=30$ years, as explained in the introduction. The acceleration factor was calculated with mean and effective temperatures and results are shown in Fig. 5.

Figure 5 shows that, as expected, the acceleration factor using the mean temperature is higher than that using the effective temperature, because the mean temperature is lower than the effective temperature. This difference is obviously enhanced by the increase of the activation energy. Consequently, equivalent time of test is shorter when using the mean temperature. Therefore, the effective temperature should be used in order to get a better lifetime assessment, and will be used hereafter in this article.

Another issue is that the acceleration factors can reach high values with high activation energy, resulting in a very low time of test that even tends to zero for colder sites. Indeed, as recalled above, a material with high activation energy is highly sensitive to temperature, and consequently only a few hours in an accelerated aging test performed at high temperature are necessary to simulate a long time on site. In conclusion, a temperature aging test is not relevant for sites with cold temperatures, and if the material has a high activation energy, the test temperature should remain relatively close to the site temperature to be representative of the degradation mechanism.

A high acceleration factor is tempting for industrial purposes, but the higher the acceleration factor, the larger the difference in the degradation mechanism under service life conditions, which makes lifetime prediction irrelevant.

\section{Basic considerations on Peck and Eyring relationships}

A well-accepted model of the humidity stress factor was proposed by $\mathrm{Peck}^{27}$ for epoxy packages for microelectronic devices and is now referred to as Peck's model. ${ }^{7,28,29}$ The corresponding relationship is given by Eq. (8), where $t_{f}$ is the time to failure, $R H$ is the relative humidity and $n$ is a kinetic parameter dependent on the material:

$t_{f}=(R H)^{n} \cdot \exp \left(\frac{E_{a(R H)}}{R \cdot T}\right)$

A second model was developed by Striny and Schelling ${ }^{30}$ based on Eyring's law and is now called Eyring's model. It is given by Eq. (9), where $C$ and $\beta$ are constants that depend on the material:

$t_{f}=C \cdot \exp \left(\frac{E_{a(R H)}}{R \cdot T}+\frac{\beta}{R H}\right)$

Both models take into account the temperature effect with an Arrhenius term. Both models are occasionally applied in parallel, and the model with the best fit of the data is then retained to predict the material behavior, ${ }^{29}$ or one model is arbitrarily chosen. 

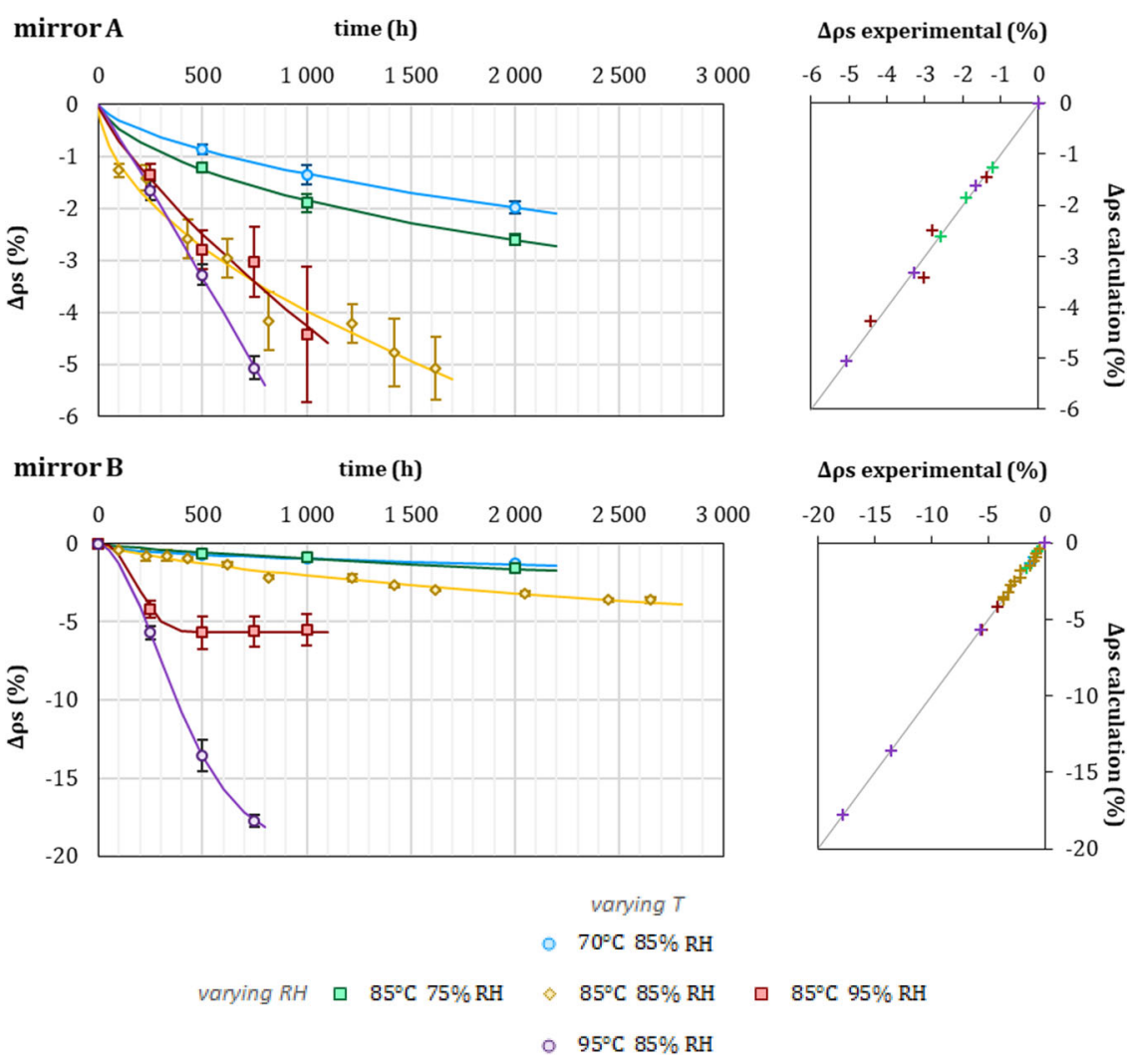

Fig. 6 Specular reflectance loss of mirrors A and B during damp heat aging tests (left). The marks are the experimental points, and the lines are the extrapolation calculated using Eq. (3). The correlation between the experimental and calculated points is shown on the right side

Experimental results of mirrors degradation

Damp heat aging tests were performed on glass mirrors $A$ and $B$ but not mirror $C$ because of a lack of samples. Similar to the results reported above, the curves were not linear, but a nice fit was obtained with the Weibull function given by Eq. (3).

The loss of specular reflectance is represented in Fig. 6 (left) as a function of time. Three tests were led varying one parameter, either the temperature or the relative humidity, and keeping the others constant (see the "Methods" section for further information). The experimental data are represented by marks, and the extrapolation calculated using Eq. (3) is represented by lines. The correlation between the experimental points and the extrapolated ones is shown for each curve on the right side of Fig. 6. The good correlation shows that the Weibull distribution function is in good agreement with experimental data on specular reflectance loss.

Degradation of mirror A at $85^{\circ} \mathrm{C} / 85 \% \mathrm{RH}$ (yellow) and $85^{\circ} \mathrm{C} / 95 \%$ $\mathrm{RH}$ (red) are close, especially if they are compared to the degradation at $85^{\circ} \mathrm{C} / 75 \% \mathrm{RH}$. This indicates that the degradation rate is hardly amplified by increasing relative humidity after a specific amount, here this amount is about $85 \% \mathrm{RH}$. At the contrary, increasing temperature still increased the degradation rate.

The degradation of mirror $\mathrm{B}$ reach a stage at $85^{\circ} \mathrm{C} / 95 \% \mathrm{RH}$. This is the shape that all curves should reach at a moment according to the Weibull Eq. (3), however, the degradation has not progress enough in other tests to see that stage.

The experimental results show that mirror $B$ is more sensitive to the damp heat test than mirror A. As mirror B was less damaged than mirror A by temperature only, it can be concluded that this result is an effect of humidity or a synergistic effect, as suggested by the rapid degradation in the $95^{\circ} \mathrm{C} / 85 \% \mathrm{RH}$ test.
Peck and Eyring numerical model parameters calculation based on experimental results

Aging tests with three temperatures at a constant relative humidity level of $85 \%$ were used to determine the thermic activation energy under humidity.

In addition, aging tests at three levels of humidity with a constant temperature of $85^{\circ} \mathrm{C}$ were used to determine the kinetic parameters $n$ and $\beta$ for Peck's (8) and Eyring's (9) models, respectively. The matrix of experiments was designed placing the $85^{\circ} \mathrm{C} / 85 \% \mathrm{RH}$ test at the center because this test is a standard test for PV modules. These conditions are also used for solar mirrors as a constant test or as a step in a cycling test. ${ }^{26}$

The kinetic parameter $n$ can be obtained by a graphical representation of $\ln \left(t_{f}\right)$ as a function of $\ln (R H)$, in which $n$ is the slope of the straight line according to Eq. (10), which is a rearrangement of Eq. (8):

$\ln \left(t_{f}\right)=n \cdot \ln (R H)+\left(\frac{E_{a(R H)}}{R \cdot T}\right)$

The kinetic parameter $\beta$ can be obtained by a graphical representation of $\ln \left(t_{f}\right)$ as a function of $1 / R H$, in which $\beta$ is the slope of the straight line according to Eq. (11), which is a rearrangement of Eq. (9):

$\ln \left(t_{f}\right)=\beta \cdot \frac{1}{R H}+\left(\frac{E_{a(R H)}}{R \cdot T}\right)+\ln (C)$

For both models, the time to failure is defined as the time of test at which the specular reflectance has lost $5 \%$.

Figure 7 shows the graphical representations based on the Arrhenius (top), Peck (middle) and Eyring (bottom) relationships. A linear fit, with a good correlation factor can be plotted in the case of mirror $A$, which indicates that the models are well appropriate. 
Arrhenius - mirror A

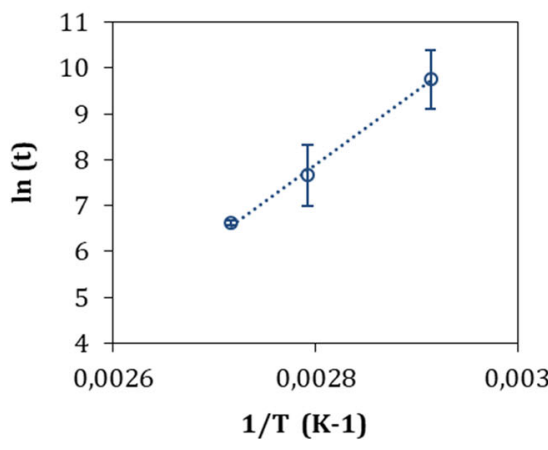

Peck - mirror A

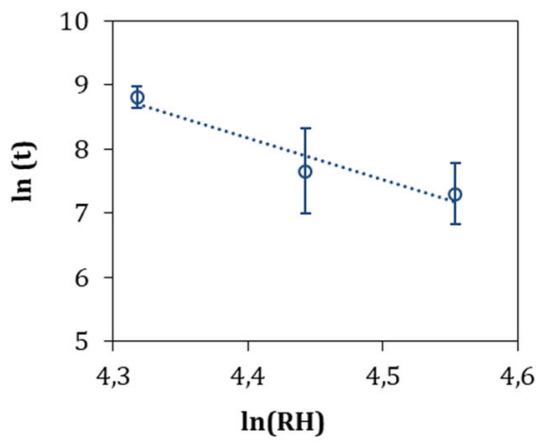

Eyring - mirror A

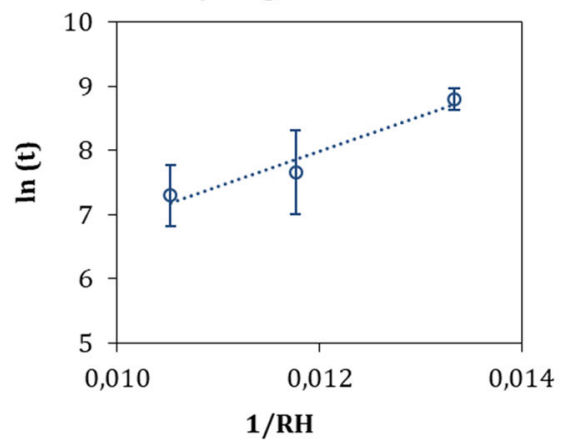

Arrhenius - mirror B

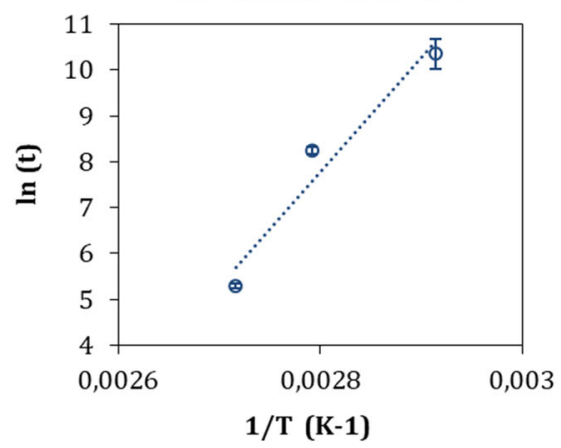

Peck - mirror B

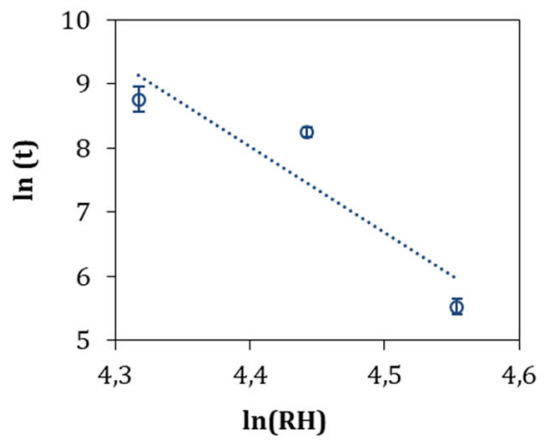

Eyring - mirror B

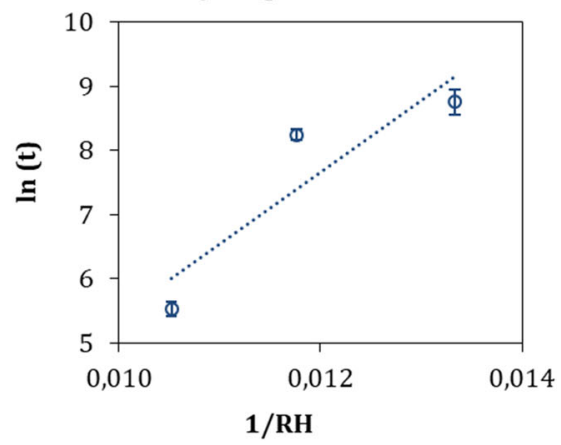

Fig. 7 Graphical representations based on Arrhenius (top), Peck (middle) and Eyring (bottom) relationships to determine respectively the apparent activation energy in the damp heat test $E_{a(R H)}$ with $\mathrm{RH}=85 \%$, and Peck's parameter $n$ and Eyring's parameter $\beta$ with $\mathrm{T}=85^{\circ} \mathrm{C}$ for mirrors $\mathrm{A}$ and $\mathrm{B}$. Error bars represent the standard deviation of experimental measurements

Table 2. Kinetic parameters for mirrors $A$ and $B$ and values from the literature ${ }^{27,29-31}$

\begin{tabular}{lccllll}
\hline & Mirror A & Mirror B & Lit. $^{30}$ & Lit. $^{29}$ & Lit. $^{27}$ \\
\hline$E_{a(R H)}(\mathrm{kJ} / \mathrm{mol})$ & $108 \pm 22$ & $162 \pm 28$ & 67 & 47 & 74 to 78 \\
$n$ & $-5.4 \pm 0.7$ & $-10.9 \pm 1.9$ & $/$ & -3.8 & -2.5 to -3 \\
$\beta(\%)$ & $544 \pm 103$ & $926 \pm 165$ & 528 & 282 & $-2.2 \pm 0.8$ \\
\hline
\end{tabular}

In the case of mirror $B$, the linear fit is not as good. However, in order to apply the full methodology, we have considered that the variations in the case of mirror B could be approximated by a straight line, despite the relatively poor correlation.

The apparent damp heat activation energies are reported in Table 2. Note that $n$ and $\beta$ can also be calculated directly from Peck and Eyring equations, respectively, but this method requires the previously calculated damp heat apparent activation energy $E_{a(R H)}$. The error on $E_{a(R H)}$ value is then reported on $n$ and $\beta$ values.
Mean results from the graphical and calculation methods are given in Table 2 for mirrors A and B with the standard deviation. Values from the literature ${ }^{27,29-31}$ are reported too for comparison with the results. There are no available data on solar mirrors, to the best of our knowledge, so the comparison was made with values established for photovoltaic modules. This comparison seems relevant to us because we considered that both devices could be seen as a functional heart, either the silver or the PV cell, protected by an envelope, either the glass and paints for mirrors or the glass for PV. 

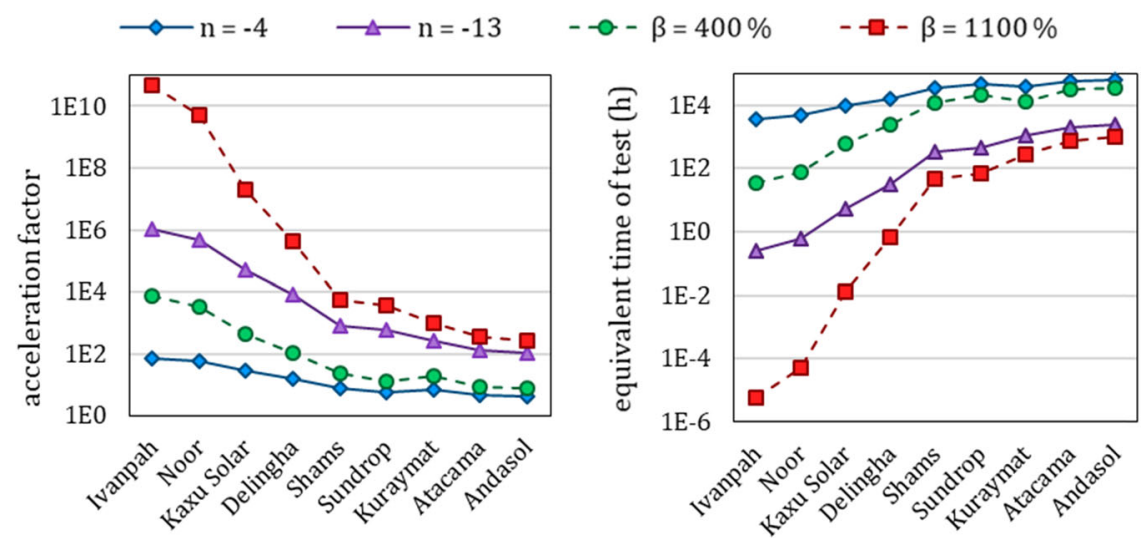

Fig. 8 Acceleration factors (left) and times of test (right) at $85 \%$ RH equivalent to 30 years on 9 sites, with $n=-4$ or -13 using Peck's model and with $\beta=400$ or $1100 \%$ using Eyring's model. Sites are sorted by increasing annual mean relative humidity

As a first conclusion, the values of the kinetic parameters are of the same order of magnitude but higher than the values reported in the literature (considering absolute value of $n$ ), in particular for mirror B. This is relevant because mirror B undergoes a dramatic failure of reflectance at high humidity that does not appear for mirror A (see Fig. 6). This suggests that the mirrors are more sensitive to damp heat test than PV modules or microelectronic devices. ${ }^{29,31}$ This finding is relevant because both of these systems are protected by packaging designed for resistance against humidity, whereas mirrors are designed for arid sites with usually low relative humidity.

Acceleration factor calculation from the numerical model The acceleration factor from Peck's model is given by Eq. (12), which is obtained by rearranging Eq. (8).

$a_{T, R H, \text { Peck }}=\left(\frac{R H_{s}}{R H_{\text {test }}}\right)^{n} \cdot \exp \left[\frac{E_{a}}{R}\left(\frac{1}{T_{s}}-\frac{1}{T_{\text {test }}}\right)\right]$

The acceleration factor from Eyring's model is given by Eq. (13), which is a rearrangement of Eq. (9).

$a_{T, R H, \text { Eyring }}=\exp \left[\frac{E_{a}}{R}\left(\frac{1}{T_{s}}-\frac{1}{T_{\text {test }}}\right)+\beta\left(\frac{1}{R H_{s}}-\frac{1}{R H_{\text {test }}}\right)\right]$

Both models include the temperature effect using an Arrhenius term. To investigate only the humidity effect, at first only the humidity terms were taken into account to calculate the acceleration factor. This simplification gives Eq. (14) for the Peck model and Eq. (15) for the Eyring model. In fact, relationship (14) has already been used in the literature. ${ }^{5}$

$a_{R H, P e c k}=\left(\frac{R H_{s}}{R H_{\text {test }}}\right)^{n}$

$a_{R H, \text { Eyring }}=\exp \left[\beta\left(\frac{1}{R H_{s}}-\frac{1}{R H_{\text {test }}}\right)\right]$

The acceleration factor was calculated for an aging test at $85 \%$ relative humidity because, as noted above, this is the value used in the damp heat test for PV devices. Two values of $n$ were chosen as examples delimiting the interval in the three previous results: -4 and -13; two values were also chosen for $\beta$ : 400 and 1100. The results are reported in Fig. 8.

The sites are arranged in order of increasing the annual humidity level in Fig. 8. A breakdown in the curves is observed at the level of Shams, which has an annual relative humidity of $50 \%$. The annual average relative humidity of Ivanpah, Noor, Kaxu Solar, and Delingha is lower than $50 \%$. The acceleration factors calculated for these sites with low humidity are very high, leading to short equivalent times of test (sometimes less than one hour).
This result indicates that the damp heat test performed under aggressive conditions of $85 \% \mathrm{HR}$ is far too destructive to obtain a relevant comparison with dry sites, especially for sensitive materials with high kinetic parameters (considering absolute value). Nevertheless, this test can be suitable for dry sites if the tested materials have a low kinetic parameter, especially using the Peck coefficient.

Calculated acceleration factors are more relevant for sites with an annual average relative humidity greater than 50\%. Among those selected for this study, these sites are Shams, Sundrop Farms, Kuraymat, Atacama and Andasol.

On the first hand, the lowest kinetic parameters of Peck and Eyring models, here taken as $n=-4$ and $\beta=400$, lead to equivalent times of test that can reach $63,000 \mathrm{~h}$. This time is obviously far too long to achieve any laboratory testing, and considerably higher than the duration recognized in the standards, which is approximately a few hundred hours. ${ }^{26,32,33}$

On the other hand, if the material has high kinetic coefficient values, here taken as $n=-13$ and $\beta=800$, the time of test can be appropriate to perform the test in laboratory.

Consequently, humidity accelerated tests should also include the effect of temperature in order to get a reasonable time of test, especially if the material of interest has a low kinetic parameter. Therefore, a relation between humidity models and temperature model will be necessary. This issue will be addressed in this article in the "Coupling stress factors" section. This experiment does not permit a choice between the two models. There is a huge difference between the Peck and Eyring values at low humidity; while the results are quite similar and tend to converge at the highest levels, namely 58 and 59\% for Atacama and Andasol, respectively. The Peck's model may be considered to give a better behavior at low humidity because the breakdown is less pronounced than for Eyring's curves.

\section{Basic considerations on Schwarzschild relationship}

Degradation due to light irradiance is usually called photodegradation. The basic law of photodegradation is the reciprocity law, which states that the same dose of light produces the same effect, independent of the dose rate. In other words, sources of light of different intensity produce the same amount of degradation under different times of exposure if multiplying the irradiance by time gives the same value. This value is called the irradiance dose or merely the dose. Thus, the product of a photochemical reaction would only depend on the total energy employed, which is the product of irradiance and time, and is independent of the two factors separately.

However, many cases of deviation of the reciprocity law have been reported in the literature. ${ }^{7,34}$ An empirical coefficient has 

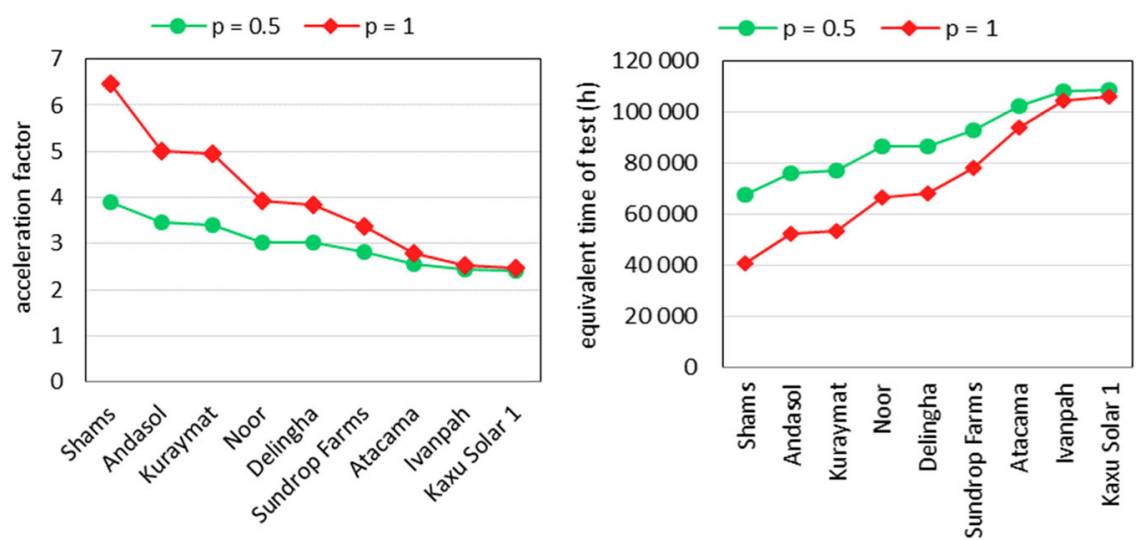

Fig. 9 Acceleration factors (left) and times of test at $65 \mathrm{~W} / \mathrm{m}^{2}$ equivalent to 30 years on site (right), calculated for 9 sites with $p=1$ and $p=0.5$ using the annual mean UV irradiance. Site are sorted by increasing irradiance

firstly been introduced by Schwarzschild ${ }^{35}$ to fit data, and since the model of the reciprocity law has been generalized with the following relationship (16): ${ }^{34}$

$D=I^{p} \times t=$ constant

where $D$ is the efficient dose, $l$ is the light intensity, $p$ is the Schwarzschild's coefficient, which is also called the $p$-coefficient, and $t$ is the time. If $p=1$, then this relationship fits the reciprocity law.

The notion of an efficient dose implies that only a part of the dose received by the product is able to cause degradation. In the case of solar mirrors, we assumed that the degradation was provoked mostly by UV irradiance, and so that the rest of the light spectrum could be disregarded. The irradiance/used hereafter is defined as the UV irradiance (with a wavelength includes between 280 and $400 \mathrm{~cm}^{-1}$ ).

Experimental results of mirrors degradation

Aging tests were performed at three levels of UV irradiance in three different chambers with a constant temperature. The results were presented in a previous work ${ }^{36}$ and are not reported hereafter. The temperature of the mirrors differed from that set in the chambers due to the method of temperature regulation of the air, which was not implemented directly on the material in two of the three chambers. Consequently, the degradation was poorly correlated with the irradiance power, and the $p$-coefficient could not be calculated.

The literature does not report data permitting the determination of a $p$ coefficient for solar glass mirrors. Consequently, two extreme values from the literature ${ }^{7,34}$ are used in the current paper: $p=0.5$ and $p=1$.

Acceleration factor calculation from the numerical model

The acceleration factor and the equivalent time of test are calculated using Eqs. (1) and (17) for the "UV dose method". UV irradiance in the aging test was set at $I_{\text {test }}=65 \mathrm{~W} / \mathrm{m}^{2}$ because this is the maximum irradiance level in the Suntest $\mathrm{XXL}+$ aging chamber (ATLAS) ${ }^{36}$. The lifetime on site is set at $t_{s}=30$ years, as explained in the introduction. Schwarzschild's coefficient is taken as either $p=0.5$ or 1 (Fig. 9).

The resulting acceleration factors for irradiance are quite weak. This is not unexpected and can easily be explained because application sites for CSP are first selected for their high irradiance levels. Thus, an irradiance aging test at room temperature would be too long even with strong intensities, and temperature must be taken into account.

Temperature plays an important role in mirror degradation and is usually used in addition to another stress factor of interest. This is particularly true for the light irradiance stress factor. Indeed, light irradiance induces a sample temperature increase that must be monitored. Ideally, the device should even permit a constant temperature while varying the intensity of irradiance. If this is not possible, it will be complicated or almost impossible to determine the kinetic parameters from the light irradiance law. Such as for temperature, it is then possible to take two extreme values from the literature data and to assess a range of lifetime under irradiance rather than a single value. This procedure has been used in this article.

As noted earlier, the acceleration factor resulting from reciprocity law is given by Eq. (17), ${ }^{7}$ considering that the degradation depends only on the efficient dose. Therefore, to achieve the same amount of degradation, the dose on-site must be equal to the dose in the test.

$a_{l}=\frac{t_{s}}{t_{\text {test }}}=\left(\frac{l_{\text {test }}}{l_{s}}\right)^{p}$

As irradiance on site varies with climatic conditions, day and night alternation and seasons, a mean value could be taken into account. Nevertheless, as the dose is assumed to be constant as the product of power and time, it seems more relevant to calculate the irradiance efficient dose using the meteorological data over a year. The p-coefficient must be determined before the calculation of the efficient dose. Then, setting the efficient dose in the test equal to the efficient dose on-site facilitates the solution of the time of test equivalent to 1 year on site according to Eq. (18):

$t_{\text {test, }, \text { year }}=\frac{D_{s}}{l_{\text {test }}^{p}}$

This time of test for 1 year can then be multiplied by the expected number of years to give the equivalent time of test, leading to relation (19).

$t_{\text {test }}=t_{s} \cdot \frac{D_{s}}{l_{\text {test }}^{p}}$

Finally, the acceleration factor can be calculated by dividing the time on site by the time of test, according to its definition by relation (1).

\section{Coupling stress factors}

Once all stress factors of interest have been studied independently, the factors must be linked together to determine a global acceleration factor.

The simplest assumption for coupling several stress factors effects is that there is no synergetic interaction between them. Accordingly, relation (20) was proposed in the literature, ${ }^{5}$ in which 


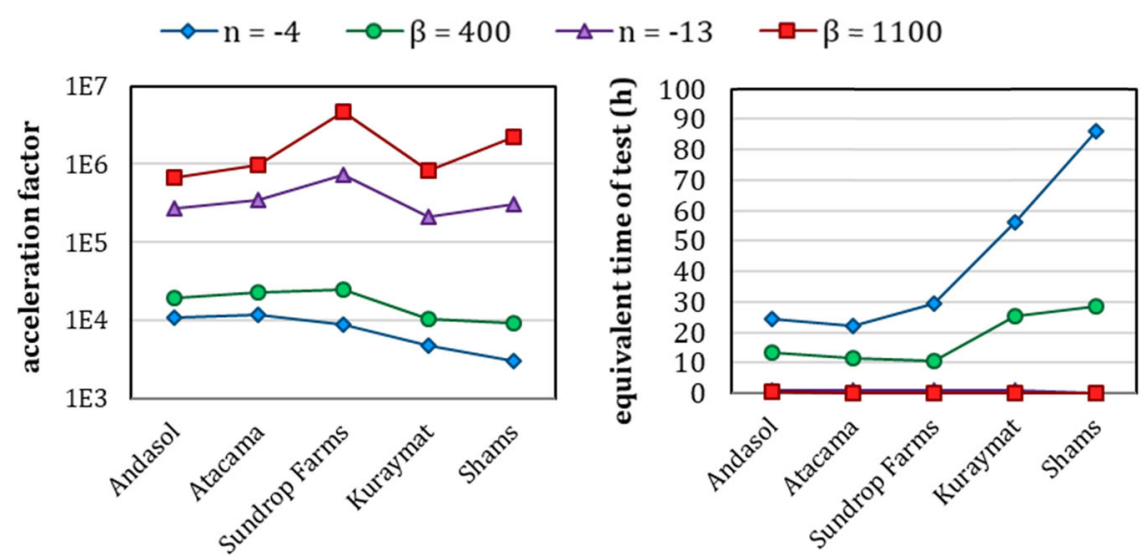

Fig. 10 Acceleration factors (left) calculated by coupling temperature and humidity and times of test (right) at $85^{\circ} \mathrm{C}$ and $85 \%$ RH equivalent to 30 years on site, calculated for 5 sites with $E_{a}=100 \mathrm{~kJ} / \mathrm{mol}$ and $n=-4$ or $\mathrm{n}=-13 ; \beta=400$ or $\beta=1100$
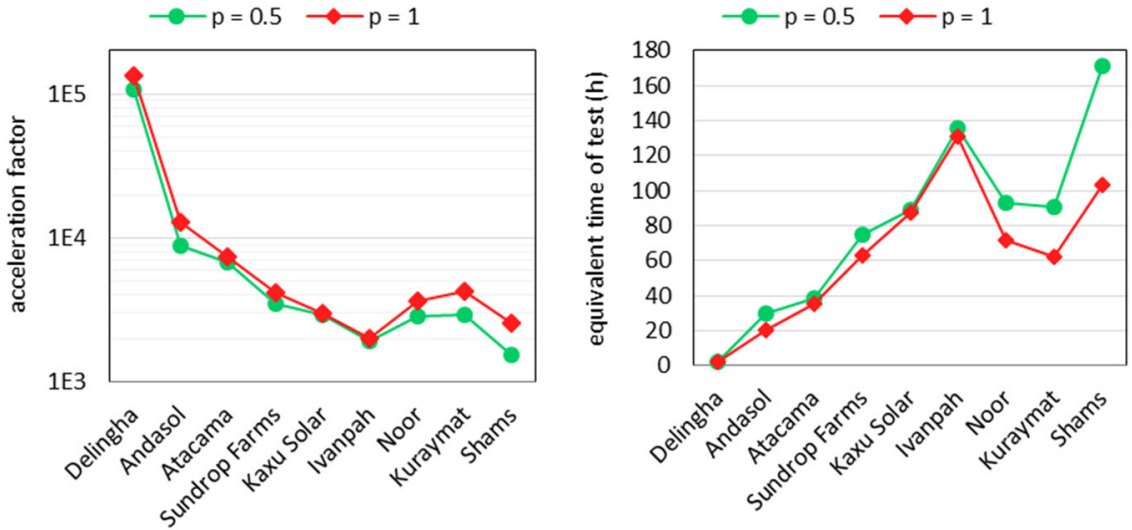

Fig. 11 Acceleration factors (left) calculated by coupling temperature and irradiance according to Eq. (20) and times of test (right) at $85^{\circ} \mathrm{C}$ and $65 \mathrm{~W} / \mathrm{m}^{2}$ equivalent to 30 years on site, calculated for 9 sites with $E_{a}=100 \mathrm{~kJ} / \mathrm{mol}$ and $p=0.5$ or 1

the global acceleration factor $A$ is the product of all acceleration factors corresponding to each stress:

$A=a_{T} \times a_{R H} \times a_{1} \times \ldots$

Acceleration factors were calculated by coupling temperature and humidity according to Eq. (20). The results on humidity models previously presented in this article show that they were inappropriate for sites with low humidity, so these sites are not considered hereafter. An aging test was set at $85^{\circ} \mathrm{C}, 85 \% \mathrm{RH}$, and $t_{s}$ was set at 30 years. The results are shown on Fig. 10 using an apparent activation energy of $100 \mathrm{~kJ} \cdot \mathrm{mol}^{-1}$ with $n=-4$ or $\mathrm{n}=$ $-13 ; \beta=400$ or $\beta=1100$. The sites are sorted by increasing mean temperature.

The damp heat test time equivalent to 30 years is less than $90 \mathrm{~h}$ according to this coupling methodology, this is a very short time. Moreover, the testing time tends to zero for high kinetic parameters. Two conclusions are possible: the coupling method leads to wrong assumptions or the damp heat test at $85^{\circ} \mathrm{C}$ and $85 \% \mathrm{RH}$ is definitely not suitable for solar mirrors.

Acceleration factors were calculated coupling temperature and irradiance according to Eq. (20), which means that the global acceleration factor is simply the product of temperature and irradiance acceleration factors, which are given by Eqs. (5) and (17), respectively. The accelerated aging test was set at $85^{\circ} \mathrm{C}$ and $65 \mathrm{~W} / \mathrm{m}^{2}$, with $t_{s}$ set at 30 years. The results are shown in Fig. 11 using an apparent activation energy of $100 \mathrm{~kJ} \cdot \mathrm{mol}^{-1}$ with $p=0.5$ or $p=1$. The sites are sorted by increasing mean temperature.

A simple product of individual acceleration factors gives very high values of the coupled acceleration factor and, consequently, irrelevantly short times of test. Indeed, this coupling predicts that less than $200 \mathrm{~h}$ would permit the simulation of 30 years on site. These values are obviously too short, and mirror aging tests performed in the chamber under temperature and irradiance do not lead to degradation so quickly compared to outdoor exposure.

The kinetic parameters of the three models were evaluated for solar mirrors in temperature and damp heat tests. The thermic apparent activation energies of the three solar mirrors were calculated according to the Arrhenius law, and the results had the expected order of magnitude. Although the differences between the values obtained for each mirror are small, the resulting acceleration factor and thus the lifetime prediction are very dependent on the precision of this value.

The calculation of an effective temperature has been performed for 9 suitable locations for CSP, CPV, and PV plants. This effective temperature is defined according to the Arrhenius law, which takes into account the exponential increase of the degradation rate with the temperature, and thus obtains a constant value that better represents the variation load of the climatic temperature than the arithmetic average.

The effective temperature is always higher than the mean temperature due to the exponential factor in the Arrhenius law. 


\begin{tabular}{|c|}
\hline Low iron glass \\
\hline Silver \\
\hline Copper \\
\hline Prime paint \\
\hline Intermediate paint \\
\hline Top paint \\
\hline
\end{tabular}
$1-4 \mathrm{~mm}$
$70-150 \mathrm{~nm}$
$30-150 \mathrm{~nm}$
$20-30 \mu \mathrm{m}$
$20-40 \mu \mathrm{m}$
$30-40 \mu \mathrm{m}$

Fig. 12 Structures of monolithic glass mirrors with usual layer thicknesses

Consequently, the acceleration factor using the effective temperature is lower than that using the mean; thus, the lifetime extrapolated is shorter. In others words, the testing time to simulate the degradation during the expected lifetime of service is longer, and thus, the degradation that occurs at the end of the ageing test could be missed using the time of test calculated with the mean temperature. Hence, the calculation of the effective temperature is important because it inhibits an overestimation of the lifetime of the material from accelerated tests. This becomes more and more important as the activation energy increases. Indeed, according to the Arrhenius law, the degradation will be faster in the case of a higher activation energy. Thus, the degradation rate at high temperatures will increase with the activation energy and increase the weight in the effective temperature calculation, which will increase too. As the activation energy has a lot of influence on the effective temperature, the one associated with the material of interest should be known precisely to gain better insights into the predicted lifetime.

The parameters of Peck's and Eyring's models were evaluated for the humidity stress factor and appeared to be in agreement with the orders of magnitude reported in the literature. The damp heat apparent activation energy in the presence of constant humidity was also assessed and differed from the thermic apparent activation energy at ambient humidity. This study highlights the difficulty of obtaining reliable values of kinetic parameters from experimental results. Future work will include material characterization to link physicochemical processes to the observed reflectance loss to associate theoretical values of the parameters with experimental assessments.

Consequently, durability studies should always give a range of predicted lifetimes using an error estimation of the kinetic parameters rather than a single value. Moreover, studies must be performed for each new mirror technology because kinetic parameters differ greatly from one technology to another (as recalled in the "Methods" section, mirrors A, B, and C all come from different manufacturers and a larger feedback from our laboratory -not reported herein- confirm this assumption).

The coupling of temperature and irradiance stress factors by multiplying the values calculated for individual stress factors leads to absurd results for mirrors, in contradiction with experimental observations, and another hypothesis should be investigated. The photothermic activation energy could be determined, as well as the damp heat activation energy, which may be different from the purely thermic activation energy.

\section{METHODS}

\section{Solar mirrors}

This article does not present an exhaustive study of mirror technologies available on the market but rather a methodology for calculating the acceleration factors at various sites around the world. The article focuses on glass mirrors because these mirrors are the most used in CSP plants. Thus, three commercially available glass mirrors are chosen as examples to make the data and results as clear as possible to understand. Samples come from three different manufacturers and are named $A, B$, and C in this article to protect confidentiality.
Solar glass mirrors have a global structure, as shown in Fig. 12. The detailed composition varies from one manufacturer to another. In particular, the back system of paints is specific to each manufacturer and can even vary from one batch to another because the solar mirrors market is not yet stabilized.

The mirrors were cut by the manufacturers to obtain square samples with a side length of $7 \mathrm{~cm}$. This size is quite small, and only the center part was considered to eliminate edge effects. All samples of a defined mirror type were taken from the same batches to avoid any effects on aging due to variation of composition or preparation. For each test, 2 or 3 samples of the same mirror were aged to obtain better representativeness of batches and to limit possible artifacts due to sampling.

\section{Characterization}

The functional property of interest for solar mirrors is their specular reflectance, which is defined as the ability to reflect a beam at the same angle as the incidence beam. The first guideline for monitoring this property was published in the framework of the SolarPACES program (Meyen et al. Parameters and method to evaluate the solar reflectance properties of reflector materials for concentrating solar power technology-Version 2.5. http://www.solarpaces.org/Tasks/Task3/reflectance_guideline.htm; June 2013). Nevertheless, the best methods for properly performing these measurements are still under discussion in the scientific community. ${ }^{37-39}$

In this research and according to the guideline from SolarPACES, the specular reflectance of samples was monitored with a portable reflectometer 15R-USB from the Device \& Services society. The reflectometer permits the measurement of specular reflectance at $\lambda=660 \mathrm{~nm}$, with an incidence angle $\theta_{\text {inc }}=15^{\circ}$. The acceptance angle was set at $\phi=25 \mathrm{mrad}$. Three measurements were performed on each sample, and the average value was taken to follow the loss of reflectance. The degradation was followed using the loss of specular reflectance $\Delta \rho_{s}$ relative to the initial state according to Eq. (21):

$\Delta \rho_{s}=\rho_{s}(t)-\rho_{s}(t=0)$

Once aging tests are performed, a performance criterion must be defined. For solar mirrors, this criterion is the given value of specular reflectance loss. Hereafter, the performance criterion was taken as $\Delta \rho s=-5 \%$ for all accelerated tests to obtain sufficient degradation while including a majority of experimental points.

\section{Accelerated tests}

All the accelerated tests were designed in order to determine kinetic parameters of the previously selected numerical models, and to assess if these models are adapted for solar mirrors lifetime prediction.

The first experiments were performed using temperature as the only stress factor. Five different temperatures with a fixed interval of $15^{\circ} \mathrm{C}$ from 70 to $130^{\circ} \mathrm{C}$ were used to evaluate the influence of temperature on the rates of specular reflectance degradation. The aim was to test the applicability of the Arrhenius law and to measure the associated activation energy. The five tests were performed in parallel in several chambers: Venticell chambers supplied by the manufacturer CTS ${ }^{\circledR}$ (Climatic \& Thermic Service), a VTU 60/60 chamber supplied by the manufacturer Weisstechnik $^{\circledast}$, and a high-temperature furnace N30/85HA supplied by Nabertherm ${ }^{\circledast}$.

The second experimental design included the coupling of humidity and temperature stress factors. This kind of test is usually called "damp heat test". A temperature higher than room temperature was required to obtain results in a reasonable time scale. Consequently, three values of relative humidity were used at a constant temperature fixed at $85^{\circ} \mathrm{C}$. However, the thermal activation energy might be different under humidity conditions than under purely thermic conditions. Therefore, the tests were also performed at three temperatures and at a constant humidity of $85 \%$ to determine the damp heat activation energy. This tests matrix was built around the classical damp heat test performed at $85{ }^{\circ} \mathrm{C}$ and $85 \% \mathrm{RH}$. This test comes from the PV field and is included in the standard which is being established for CSP mirrors. The following chambers were used: LHU-114 supplied by the manufacturer ESPEC Corp ${ }^{\circledast}$, VC0018 supplied by Vötsch ${ }^{\circledast}$ and WKL 100-40 supplied by Weisstechnik ${ }^{\circledR}$.

The tests conditions are summarized in Table 3. All tests were performed in the dark.

\section{Sites selection}

The acceleration factor is not universal and should always be calculated for a given site. To apply the complete methodology, different sites must be 
chosen as examples. To ensure relevance for solar mirrors, the selected sites were locations of operational CSP plants or projects under construction. Our second criterion was high thermal amplitude variation

\begin{tabular}{|c|c|c|}
\hline Temperature $\left({ }^{\circ} \mathrm{C}\right)$ & Humidity (\%) & Chamber \\
\hline 70 & Ambient & Venticell \\
\hline 85 & Ambient & Venticell \\
\hline 100 & Ambient & Venticell \\
\hline 115 & Ambient & VTU $60 / 60$ \\
\hline 130 & Ambient & $\mathrm{N} 30 / 85 \mathrm{HA}$ \\
\hline 85 & 75 & LHU-114 \\
\hline 70 & 85 & LHU-114 \\
\hline 85 & 85 & VC0018 \\
\hline 95 & 85 & WKL100-40 \\
\hline 85 & 95 & VC0018 \\
\hline
\end{tabular}

during the years of the study, as determined using Meteonorm ${ }^{\circledast} 7.2$ software (Company, Meteotest Meteonorm 7: Global Meteorological Database for Solar Energy and Applied Climatology. (2015)). Finally, the sites were chosen to give a global distribution, as shown on Fig. 13 which represents the world map of Köppen-Geiger Climate classification. ${ }^{2}$ This is a limited sample of locations used as an example for the methodology; however, this work aims to be applied to many other sites of interest.

Meteorological data including temperature, solar irradiance, and humidity, among other parameters, were extracted using Meteonorm ${ }^{\circledast}$ software. This software reports measurements from weather stations located all around the world and from five geostationary satellites. A typical year is then calculated by taking the mean over the years of data measurements. The periods are 1991-2010 for irradiance data and 2000-2009 for humidity and temperature data. Moreover, data can be extracted for any location because the software uses interpolation models between the closest weather stations. The yearly mean weather data for the selected sites are shown in Table 4.

Figure 14 shows the frequency of temperatures in hours per year for each selected site, and all graphs are plotted using the same temperature and frequency scale. The interval of temperature was set to be $1^{\circ} \mathrm{C}$. This diversity of the temperature diagram over the year was chosen to explore the impact on the effective temperature calculation.

Figure 15 shows the frequency of relative humidity in hours per year for each selected site, and all graphs are plotted using the same relative

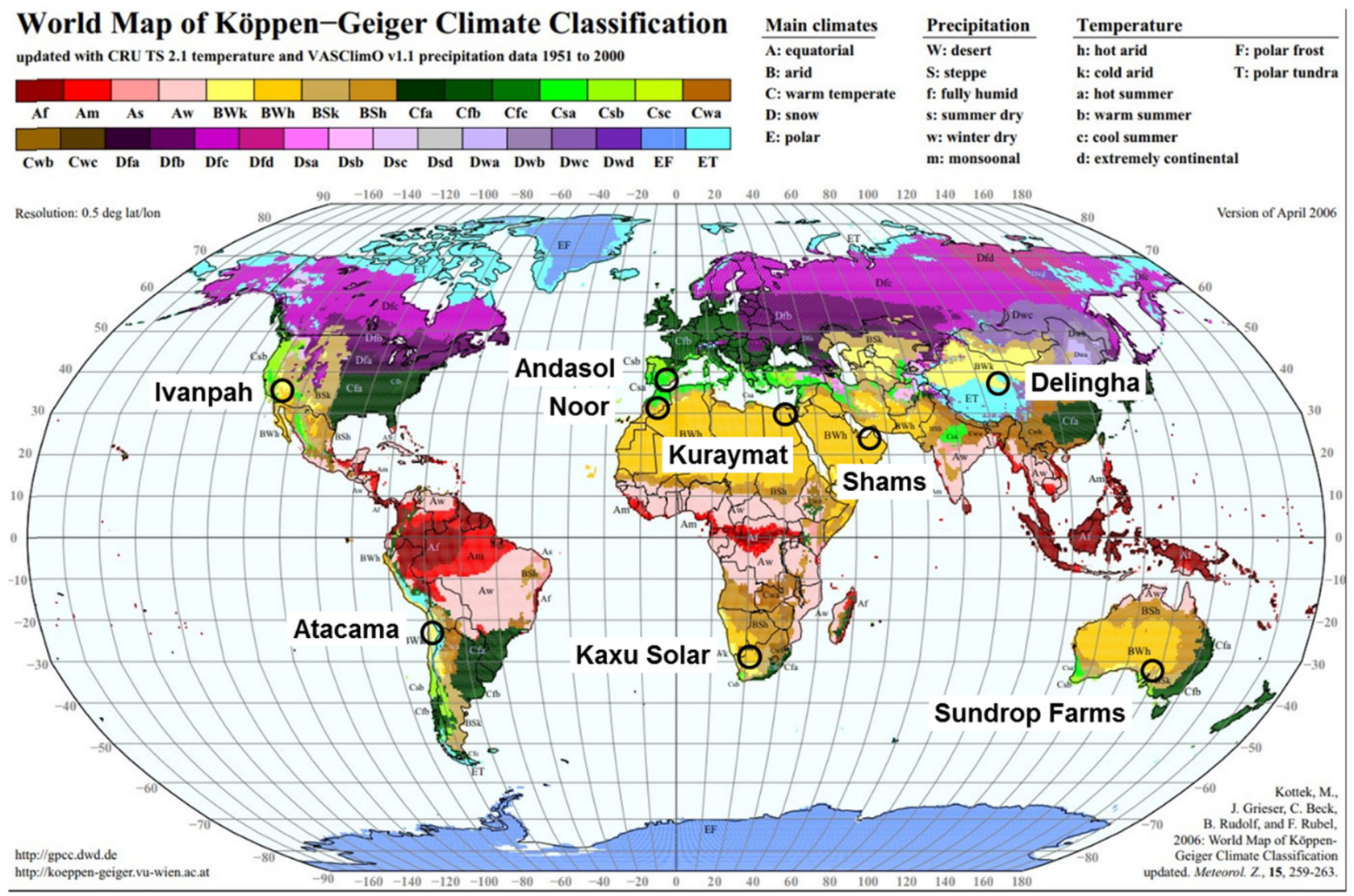

Fig. 13 Location of the sites on the world map of Köppen-Geiger Climate classification ${ }^{2}$

Table 4. Yearly mean meteorological data: temperature (T), UV irradiance (I) and relative humidity (RH) for the selected sites from Meteonorm software

\begin{tabular}{llllllllll}
\hline & Andasol & Atacama & Delingha & Ivanpah & Kaxu Solar & Kuraymat & Noor & Shams & Sundrop \\
\hline $\mathbf{T ~}\left({ }^{\circ} \mathbf{C}\right)$ & 12.7 & 16.3 & -5.9 & 19.7 & 19.3 & 22.6 & 19.9 & 28.2 \\
$\mathbf{I}\left(\mathbf{W} / \mathbf{m}^{\mathbf{2}}\right)$ & 13 & 23.3 & 16.9 & 25.9 & 26.2 & 13.2 & 16.5 & 10.1 \\
$\mathbf{R H}(\%)$ & 59 & 58 & 42 & 29 & 36 & 55 & 31 & 50 & 51 \\
\hline
\end{tabular}



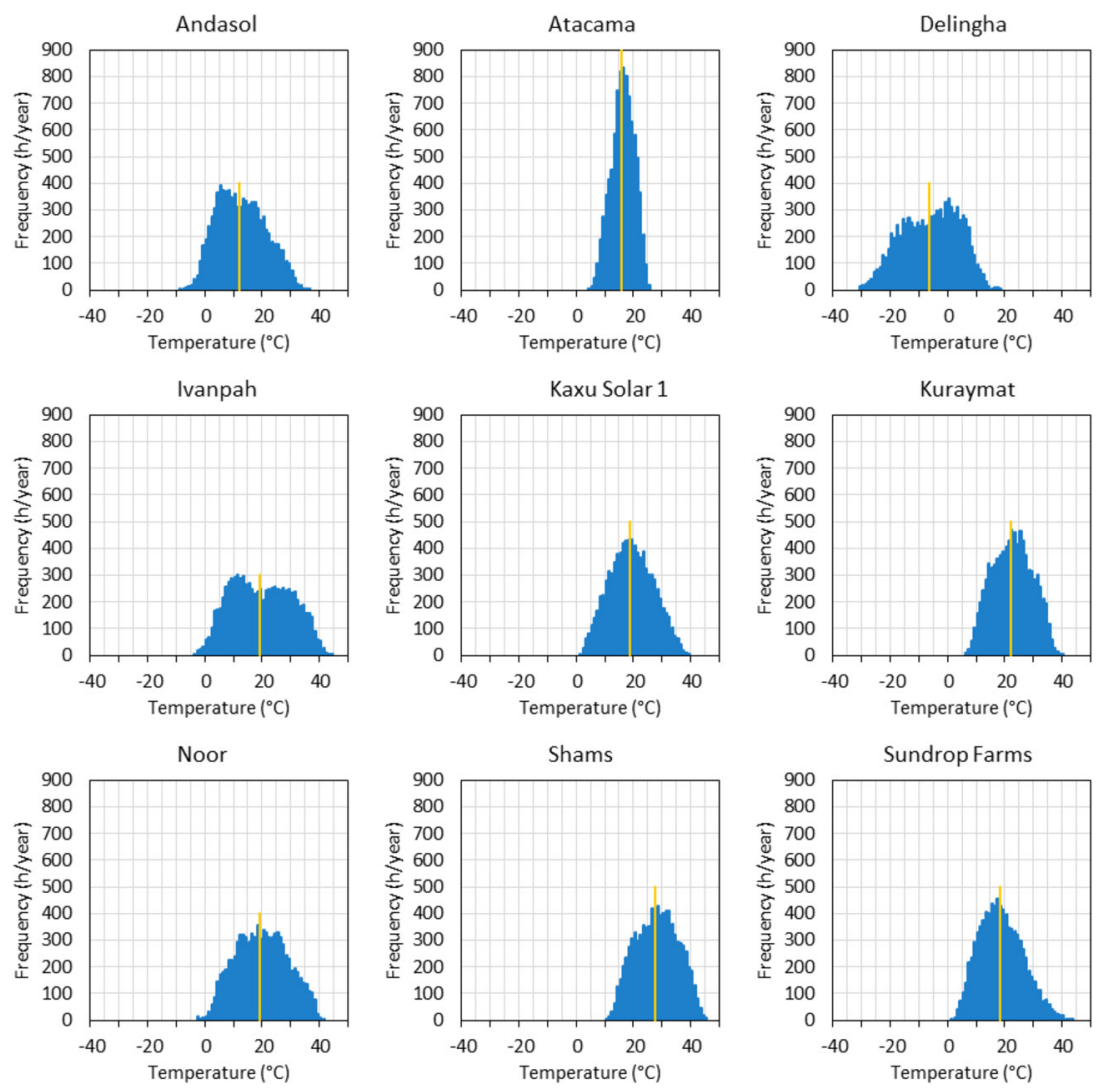

Fig. 14 Temperature frequency in hours per year for the 9 sites, the yellow straight line highlights the mean temperature
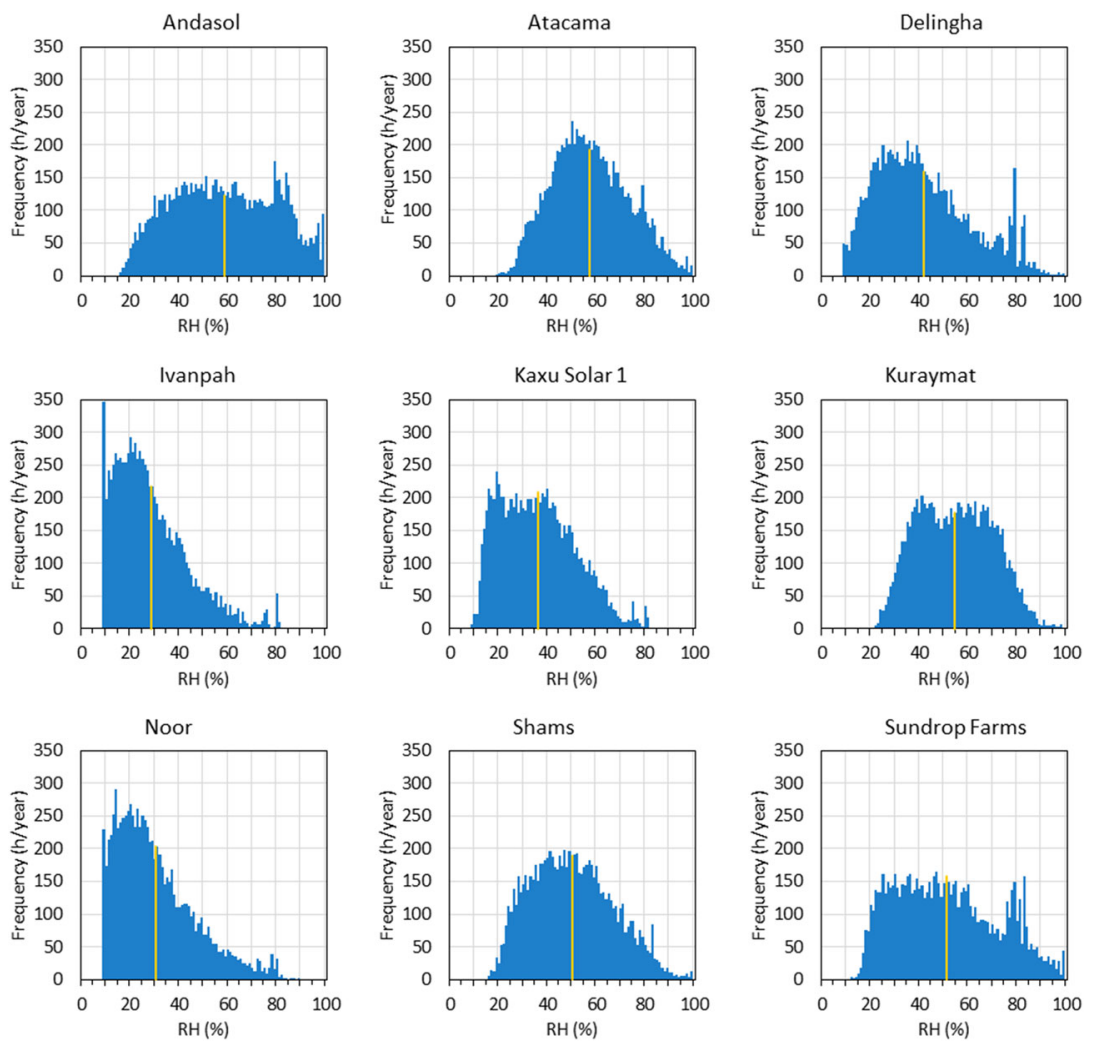

Fig. 15 Relative humidity frequency in the range 10-100\% in hours per year for the 9 sites. The yellow straight line highlights the average annual relative humidity (data obtained from Meteonorm ${ }^{\circledast} 7.2$ software (Company, Meteotest Meteonorm 7: Global Meteorological Database for Solar Energy and Applied Climatology. (2015) 
humidity and frequency scale. The interval of relative humidity was set to be $1 \%$.

\section{DATA AVAILABILITY}

The data that support the findings of this study are available from the corresponding author upon reasonable request.

\section{ACKNOWLEDGEMENTS}

The research leading to this article received French national funding from Agence Nationale de la Recherche as part of the program Investissements d'Avenir ( $n^{\circ}$ ANR11-EQPX-0014) and funding from the Auvergne region.

\section{AUTHOR CONTRIBUTIONS}

All authors provided substantial contributions to conception of the research performed. C.A and O.R. performed the acquisition of the data. The analysis of the data and the interpretation of the results were discussed with J-L.G. and S.T. C.A. and O.R. drafted and J-L.G. and S.T. revised the manuscript and approved the final version. The four authors are accountable for the accuracy and integrity of the data and their interpretation.

\section{ADDITIONAL INFORMATION}

Competing interests: The authors declare no competing interests.

Publisher's note: Springer Nature remains neutral with regard to jurisdictional claims in published maps and institutional affiliations.

\section{REFERENCES}

1. Mehos, M. et al. Concentrating Solar Power Gen3 Demonstration Roadmap. Technical Report NREL/TP-5500-67464 (2017).

2. Kottek, M., Grieser, J., Beck, C., Rudolf, B. \& Rubel, F. World map of Köppen-Geiger climate classification uptdated. Meteorol. Z. 15, 259-263 (2006).

3. Girard, R., Delord, C., Disdier, A. \& Raccurt, O. CritiCal Constraints Responsible To Solar Glass Mirror Degradation. Energy Procedia 69, 1519-1528 (2015).

4. Schissel, P., Jorgensen, G., Kennedy, C. \& Goggin, R. Silvered-PMMA reflectors. Sol. Energy Mater. Sol. Cells 33, 183-197 (1994).

5. Carlsson, B., Jorgensen, G. \& Köhl, M. Chapter 1.1 - Introduction to the Performance and Durability Assessment of Optical Materials for Solar Thermal Systems in Performance and Durability Assessment (eds Köhl, M., Jorgensen, G., Carlsson, B. \& Czanderna, A. W.) 3-16 (Elsevier Science, 2004).

6. García-Segura, A., Fernández-García, A., Ariza, M. J., Valenzuela, L. \& Sutter, F. Durability studies of solar reflectors: A review. Renew. Sustain. Energy Rev. 62, 453-467 (2016).

7. Avenel, C., Raccurt, O., Gardette, J.-L. \& Therias, S. Review of accelerated ageing test modelling and its application to solar mirrors. Sol. Energy Mater. Sol. Cells 186, 29-41 (2018).

8. Czanderna, A. W. \& Schissel, P. Specularity and stability of silvered polymers. Sol. Energy Mater. Sol. Cells 14, 341-356 (1986).

9. Brogren, M., Helgesson, A., Karlsson, B., Nilsson, J. \& Roos, A. Optical properties, durability, and system aspects of a new aluminium-polymer-laminated steel reflector for solar concentrators. Sol. Energy Mater. Sol. Cells 82, 387-412 (2004).

10. Baharoon, D. A., Rahman, H. A., Omar, W. Z. W. \& Fadhl, S. O. Historical development of concentrating solar power technologies to generate clean electricity efficiently: A review. Renew. Sustain. Energy Rev. 41, 996-1027 (2015).

11. Fuqiang, W. et al. Progress in concentrated solar power technology with parabolic trough collector system: a comprehensive review. Renew. Sustain. Energy Rev. 79, 1314-1328 (2017).

12. Weinstein, L. A. et al. Concentrating solar power. Chem. Rev. 115, 12797-12838 (2015).

13. Haillant, O., Dumbleton, D. \& Zielnik, A. An Arrhenius approach to estimating organic photovoltaic module weathering acceleration factors. Sol. Energy Mater. Sol. Cells 95, 1889-1895 (2011).

14. Dia, F. et al. Model associated with the study of the degradation based on the accelerated test: a literature review. Open J. Appl. Sci. 6, 49-63 (2016).

15. Escobar, L. A. \& Meeker, W. Q. A review of accelerated test models. Stat. Sci. 21, 552-577 (2006).

16. Weibull, W. A statistical distribution function of wide applicability. J. Appl. Mech. 18, 293-297 (1951).

17. Prendergast, J., O'Driscoll, E. \& Mullen, E. Investigation into the correct statistical distribution for oxide breakdown over oxide thickness range. Microelectron. Reliab. 45, 973-977 (2005).
18. Weis, E. A., Caldararu, D., Snyder, M. M. \& Croitoru, N. Reliability evaluation and prediction for silicon photodetectors. IEEE Trans. Reliab. 37, 14-23 (1988).

19. $\mathrm{Yu}, \mathrm{H} .-\mathrm{F}$. Designing an accelerated degradation experiment with a reciprocal Weibull degradation rate. J. Stat. Plan. Inference 136, 282-297 (2006).

20. Celina, M. Review of polymer oxidation and its relationship with materials performance and lifetime prediction. Polym. Degrad. Stab. 98, 2419-2429 (2013).

21. Kurtz, S. et al. Evaluation of high-temperature exposure of rack-mounted photovoltaic modules. 34th IEEE Photovoltaic Specialists Conference (PVSC) 2399-2404 (2009).

22. McMahon, T. J. Accelerated testing and failure of thin-film PV modules. Prog. Photovolt.: Res. Appl. 12, 235-248 (2004).

23. Tencer, M., Moss, J. S. \& Zapach, T. Arrhenius average temperature: the effective temperature for non-fatigue wearout and long term reliability in variable thermal conditions and climates. IEEE Trans. Compon. Packag. Technol. 27, 602-607 (2004).

24. Brunold, S. et al. Accelerated life testing of solar absorber coatings: Testing procedure and results. Sol. Energy 68, 313-323 (2000).

25. Carlsson, B., Moller, K., Köhl, M., Frei, U. \& Brunold, S. Qualification test procedure for solar absorber surface durability. Sol. Energy Mater. Sol. Cells 61, 255-275 (2000).

26. Fernández-García, A. et al. Accelerated aging test of solar reflectors according to the new AENOR Standard - results of a round Robin Test. Conf.: Sol. 2017: Int. Conf. Conc. Sol. Power Chem. Energy Syst. Novemb. 2018 AIP Conf. Proc. 2033, 230003 (2018).

27. Peck, D. S. Comprehensive Model for Humidity Testing Correlation. 24th International Reliability Physics Symposium 44-50 (1986).

28. Carlsson, B. et al. The applicability of accelerated life testing for assessment of service life of solar thermal components. Sol. Energy Mater. Sol. Cells 84, 255-274 (2004).

29. Park, N. C., Oh, W. W. \& Kim, D. Effect of temperature and humidity on the degradation rate of multicrystalline silicon photovoltaic module. Int. J. Photo. 2013, 925280 (2013).

30. Striny, K. \& Schelling, A. Reliability evaluation of aluminum-metallized MOS dynamic RAM's in plastic packages in high humidity and temperature environments. IEEE Trans. Compon., Hybrids, Manuf. Technol. 4, 476-481 (1981).

31. Kimball, G. M., Yang, S., Saproo, A. Global acceleration factors for damp heat tests of PV modules. Conference: 2016 IEEE 43rd Photovoltaic Specialists Conference (PVSC) 101-105 (2016).

32. Sutter, F., Fernandez-Garcia, A., Wette, J. \& Heller, P. Comparison and evaluation of accelerated aging tests for reflectors. Energy Procedia 49, 1718-1727 (2014).

33. Wette, J., F Sutter, F., Fernández-García, A., Ziegler, S. \& Dasbach, R. Comparison of degradation on aluminium reflectors for solar collectors due to outdoor exposure and accelerated aging. Energies 9, 916 (2016).

34. Martin, J. W., Chin, J. W. \& Nguyen, T. Reciprocity law experiments in polymeric photodegradation: a critical review. Prog. Org. Coat. 47, 292-311 (2003).

35. Schwarzschild, K. On the deviations from the law of reciprocity for bromide of silver gelatine. Astrophys. J. 11, 89-91 (1900).

36. Avenel, C., Gardette, J. L., Therias, S., Disdier, A. \& Raccurt, O. Accelerated aging test of solar mirrors: comparison of different UV chambers. AIP Conf. Proc. 1850, 130001 (2017).

37. Arancibia-Bulnes, C. A., Pea-Cruz, M. I., Mutuberra, A., Diaz-Uribe, R. \& SanchezGonzalez, M. A survey of methods for the evaluation of reflective solar concentrator optics. Renew. Sustain. Energy Rev. 69, 673-684 (2017).

38. Sansom, C., Fernandez-Garcia, A., King, P., Sutter, F. \& Garcia Segura, A. Reflectometer comparison for assessment of back-silvered glass solar mirrors. Sol. Energy 155, 496-505 (2017).

39. Sutter, F., Montecchi, M., Dalhen, H., Fernadez, A. \& Garcia, M. R. The effect of incidence angle on the reflectance of solar mirrors. Sol. Energy Mater. Sol. Cells 176, 119-133 (2018).

Open Access This article is licensed under a Creative Commons Attribution 4.0 International License, which permits use, sharing, adaptation, distribution and reproduction in any medium or format, as long as you give appropriate credit to the original author(s) and the source, provide a link to the Creative Commons license, and indicate if changes were made. The images or other third party material in this article are included in the article's Creative Commons license, unless indicated otherwise in a credit line to the material. If material is not included in the article's Creative Commons license and your intended use is not permitted by statutory regulation or exceeds the permitted use, you will need to obtain permission directly from the copyright holder. To view a copy of this license, visit http://creativecommons. org/licenses/by/4.0/.

(c) The Author(s) 2019 